WM-00-111

\title{
A LOT OF FLAVOR PHYSICS FROM A LITTLE SYMMETRY*
}

\author{
ALFREDO ARANDA and CHRISTOPHER D. CARONE \\ Nuclear and Particle Theory Group, College of William and Mary \\ Williamsburg, Virginia 23187-8795, USA \\ RICHARD F. LEBED \\ Department of Physics and Astronomy, Arizona State University \\ Tempe, Arizona 85287-1504, USA
}

October 2000

\begin{abstract}
Recent neutrino parameter measurements place increasingly stringent constraints on acceptable supersymmetric theories of flavor. A very fruitful approach is the application of non-Abelian discrete gauged flavor symmetries $G_{f}$. We discuss a highly successful model using $G_{f}=T^{\prime} \times Z_{3}$, where $T^{\prime}$ is a non-Abelian group based on the symmetries of a tetrahedron. This model reproduces the basic features of the U(2) model, and also predicts neutrino masses and mixing angles consistent with either the SMA or LMA scenarios.
\end{abstract}

The family replication problem and the strongly hierarchical pattern of fermion masses and mixing angles has remained one of the more intractable problems in particle physics. Any acceptable flavor theory must explain why, for example, $m_{t} / m_{e} \approx$ 350,000 while $\left|V_{u b}\right| \approx 3 \times 10^{-3}$. The situation has become even more interesting with the observation of nonzero neutrino masses and mixingst that do not appear to follow the same hierarchies as in the charged fermion sector. Indeed, the combined data of existing experiments (omitting LSNDE) suggests a large mixing angle due to the atmospheric $\nu$ deficit,

$$
\sin ^{2} 2 \theta_{23}>0.8, \quad 10^{-3} \mathrm{eV}^{2} \leq \Delta m_{23}^{2} \leq 10^{-2} \mathrm{eV}^{2},
$$

and a solar $\nu$ deficit explained by either the small mixing angle (SMA) MSW solution

$$
2 \times 10^{-3} \leq \sin ^{2} 2 \theta_{12} \leq 10^{-2}, \quad 4 \times 10^{-6} \mathrm{eV}^{2} \leq \Delta m_{12}^{2} \leq 10^{-5} \mathrm{eV}^{2},
$$

or the (now somewhat more favored) large mixing angle (LMA) MSW solution

$$
\sin ^{2} 2 \theta_{12}>0.7, \quad 6 \times 10^{-6} \mathrm{eV}^{2} \leq \Delta m_{12}^{2} \leq 5 \times 10^{-5} \mathrm{eV}^{2} .
$$

with three active neutrino species.

We consider the possibility that a spontaneously-broken horizontal symmetry $G_{f}$ may explain the totality of fermion mass and mixing angle data. We work in the context of supersymmetry (SUSY), which renders the hierarchy between the scale of flavor physics and the weak scale stable against radiative corrections.

*Talk presented by R.F. Lebed at DPF 2000, Ohio State Univ., Columbus, OH, 9-12 Aug. 2000. 
Flavor-changing neutral current (FCNC) effects, which are present in generic SUSY models, can be brought under control by the same symmetry $G_{f}$ if it leads to a near mass degeneracy between the first two generations of squarks or sleptons in the low-energy effective theory. We require this to be the case.

We advocate the study of discrete gauge symmetries for $G_{f}$. It has long been argued 3 that global symmetries are violated by quantum gravity effects and may therefore be inconsistent as fundamental symmetries of nature. Alternately, continuous gauge symmetries in the context of SUSY tend to give rise to excessive FCNC's through D-term interactions. 1 Discrete gauge symmetries avoid both of these pitfalls. Theories with global and gauged discrete symmetries differ in that there is information contained in topological defects found only in the latter 0.6 For modelbuilding purposes, the relevant low-energy constraint for discrete gauge symmetries is an anomaly cancellation copdition linear in $G_{f}$ and quadratic in non-Abelian Standard Model gauge groups. 0 Our model satisfies this condition.

The central feature of discrete groups is a finite number of inequivalent irreducible matrix representations (reps). Discrete groups tend to have a rich representation structure, which opens many possibilities for assigning quantum numbers in model building. Reps of dimension $>1$ appear only in non-Abelian groups. In particular, doublets are used to impose the near-degeneracy of sparticle masses in the first two generations, while the large value of $m_{t}$ suggests that the third generation is distinguished: a $\mathbf{2} \oplus \mathbf{1}$ structure.

A model based on the continuous group $G_{f}=\mathrm{U}(2)$ is particularly successful in reproducing all observed charged fermion masses and mixings, especially when combined with an SU(5) GUT. The Yukawa matrix textures are given by

$$
Y_{D} \sim Y_{L} \sim\left(\begin{array}{ccc}
0 & \epsilon^{\prime} & 0 \\
-\epsilon^{\prime} & \epsilon & \epsilon \\
0 & \epsilon & 1
\end{array}\right), \quad Y_{U} \sim\left(\begin{array}{ccc}
0 & \epsilon^{\prime} \epsilon & 0 \\
-\epsilon^{\prime} \epsilon & \epsilon^{2} & \epsilon \\
0 & \epsilon & 1
\end{array}\right)
$$

where the symmetry is broken at two scales, $U(2) \stackrel{\epsilon}{\longrightarrow} U(1) \stackrel{\epsilon^{\prime}}{\longrightarrow}$ nothing, with $\epsilon \approx 0.02$ and $\epsilon^{\prime} \approx 0.004$. In order to produce these textures, the $\mathrm{U}(2)$ model requires $\mathbf{1}, \mathbf{2}$ and $\mathbf{3}$ reps and the multiplication rule $\mathbf{2} \otimes \mathbf{2}=\mathbf{3} \oplus \mathbf{1}$.

One finds 9 that the smallest group satisfying the same conditions is $T^{\prime}$, the group of proper rotations leaving a regular tetrahedron invariant in the $\mathrm{SU}(2)$ double covering of $\mathrm{SO}(3)$. Moreover, including an extra $Z_{3}$ factor, so that $G_{f}=T^{\prime} \times Z_{3}$, is the minimal extension needed to reproduce $\mathrm{U}(2)$ model textures and satisfy discrete anomaly cancellation conditions. Additionally, the rich representation structure of $T^{\prime}$ allows for neutrinos to be placed in different reps than the charged fermions, which is the origin of different hierarchies in the two sectors. The symmetry breaking is $T^{\prime} \times Z_{3} \stackrel{\epsilon}{\longrightarrow} Z_{3}^{\text {diag }} \stackrel{\epsilon^{\prime}}{\longrightarrow}$ nothing, and with the charge assignments presented in 目, the light neutrino mass matrix (via the seesaw mechanism) is

$$
M_{L L} \sim\left(\begin{array}{ccc}
\left(\epsilon^{\prime} / \epsilon\right)^{2} & \epsilon^{\prime} / \epsilon & \epsilon^{\prime} / \epsilon \\
\epsilon^{\prime} / \epsilon & 1 & 1 \\
\epsilon^{\prime} / \epsilon & 1 & 1
\end{array}\right)
$$


Table 1. Data and fit values for the LMA scenario in the $T^{\prime} \times Z_{3}$ model ( $\nu$ sector).

\begin{tabular}{lll} 
Observable & Expt. value & Fit value \\
\hline$\Delta m_{23}^{2} / \Delta m_{12}^{2}$ & $20-1670$ & 376 \\
$\sin ^{2} 2 \theta_{12}$ & $>0.8$ & 0.88 \\
$\sin ^{2} 2 \theta_{23}$ & $>0.8$ & 0.83 \\
$\sin ^{2} 2 \theta_{13}$ & $<0.18$ & 0.10 \\
\hline
\end{tabular}

The 23 mixing angle is clearly $O(1)$; since $\epsilon^{\prime} / \epsilon=O(1 / 5)$ is neither especially large nor small, adjustment of the implicit $O(1)$ coefficients characteristic of an effective field theory allows for either LMA or SMA solutions for $\theta_{12}$.

We present in Table 1 a fit to the observable neutrino mass and mixing parameters for LMA; the full fit also includes the charged fermion parameters, but is excellent and hence omitted for brevity. A similarly excellent SMA fit appears in 1 . The unknown $O(1)$ coefficients $c_{i}$ are constrained to lie approximately in the range $1 / r \leq\left|c_{i}\right| \leq r$ by adding terms to the $\chi^{2}$ function of form $\Delta \chi_{i}^{2}=\left(\ln \left|c_{i}\right| / \ln r\right)^{2}$; for this fit, $r=3$. One finds $\chi_{\text {min }}^{2}=11.8$, smaller than the total number of fermion observables (16), which we argue 11 is the figure of merit.

The explosion of neutrino data and better bounds on rare FCNC processes will provide ever more precise clues as to the nature of flavor physics. We have seen how well a rather small discrete gauge group can accommodate all of the currently measured observables and anticipate the refinement of such models in the future.

\section{Acknowledgments}

A.A. and C.D.C. thank for support the National Science Foundation under Grant No. PHY-9900657, and the Jeffress Memorial Trust under Grant No. J-532. RFL thanks the Department of Energy for support under Grant No. DE-AC05-84ER40150 and the U. Maryland TQHN Group for their hospitality.

\section{References}

1. See J.N. Bahcall, P.I. Krastev, and A.Y. Smirnov, Phys. Rev. D 58 (1998) 096016.

2. LSND Collaboration, C. Athanassopoulos et al., Phys. Rev. Lett. 81 (1998) 1774.

3. S. Coleman, Nucl. Phys. B310 (1988) 643; S. Giddings and A. Strominger, ibid. B307 (1988) 854; G. Gilbert, ibid. B328 (1989) 159.

4. Y. Kawamura, H. Murayama, and M. Yamaguchi, Phys. Rev. D 51 (1995) 1337.

5. L.M. Krauss and F. Wilczek, Phys. Rev. Lett. 62 (1989) 1221.

6. T. Banks, Nucl. Phys. B323 (1989) 90.

7. L.E. Ibáñez and G.G. Ross, Phys. Lett. B 260 (1991), 291; T. Banks and M. Dine, Phys. Rev. D 45 (1992) 1424.

8. R. Barbieri, G. Dvali, and L.J. Hall, Phys. Lett. B 377 (1996) 76; R. Barbieri, L.J. Hall, and A. Romanino, ibid. B 401 (1997) 47; R. Barbieri, L.J. Hall, S. Raby, and A. Romanino, Nucl. Phys. B493 (1997) 3.

9. A. Aranda, C.D. Carone, and R.F. Lebed, Phys. Lett. B 474 (2000) 170.

10. A. Aranda, C.D. Carone, and R.F. Lebed, Phys. Rev. D 62 (2000) 016009. 